\title{
Negotiating Stereotypes of Older Adults through Avatars
}

\author{
Romina Carrasco, Steven Baker, Jenny Waycott, Frank Vetere \\ Interaction Design Lab, School of Computing and Information Systems \\ The University of Melbourne, Australia \\ rcarrasco@student.unimelb.edu.au, steven.baker, jwaycott, f.vetere [@unimelb.edu.au]
}

\begin{abstract}
Virtual Avatars can bring opportunities for enjoyment, social participation and exploration of identities. However, the configuration of avatar creation software may marginalise some groups of users due to them reinforcing social stereotypes that privilege youth and beauty, rather than representing the broader variety of human identities. Older adults are one group who may be disadvantaged with respect to avatars as avatar studies have typically focused on younger users. Considering that older populations are growing and that their participation in virtual environments is increasing, it is timely to investigate older adults' preferences in relation to avatars. We conducted a study with 23 participants (70+ years old) to understand the representational requirements of older adults when creating a humanoid virtual avatar. Our findings demonstrate that older adults are negotiating ageing stereotypes when creating a virtual body. These negotiations of body appearances range from: the Actual Avatar that by mirroring the self suggests an acceptance of the ageing body; the Vibrant Avatar that is idealising the physical condition of the self; the Other Avatar, that aims to explore other identities; and the Companion Avatar that creates another persona as company. These findings highlight that older adults have specific representational requirements when designing virtual avatars.
\end{abstract}

\section{CCS CONCEPTS}

- Human-centered computing $\rightarrow$ Empirical studies in interaction design • Human-centered computing $\rightarrow$ Graphical user interfaces

\section{KEYWORDS}

Virtual avatars, older adults, aging stereotypes, identity, selfrepresentation.

\section{INTRODUCTION}

Stereotypes depict older adults as reluctant users of digital technologies. Recent research, however suggests that new technologies are being embraced by a growing proportion of older adults. Older adults are now blogging [5], using social media [7], and playing online games [27]. These online games are not limited to card games and word puzzles - as stereotypes would suggest - but extend to role-playing games that take place in virtual environments [27]. A recent high profile example is the story of 'Gamer Grandma', an 81-year-old grandma who plays Skyrim, a role-playing video game in which the player embodies fantasy characters to join a fictional world [22].

In these virtual environments, players are represented by virtual avatars, which are typically animated (not static) and move in response to controls issued by the user in real time. The customisation of an avatar is usually the first step when entering a virtual environment for the first time. The challenge for older adults in these spaces, however, is that there may be limitations in the options available for creating and customising avatars, making it difficult to represent the ageing body. Avatar creation software tends to mirror societal stereotypes which value youth and beauty over more diverse representations [41]. This can limit diversity in online spaces, something players are becoming concerned about. A recent survey of digital game players in Australia found that $65 \%$ of those surveyed said they believed there was a need for more diversity of age portrayal of characters in games [4].

This raises questions about whether avatars currently respond to the needs of older users, or indeed whether older adults want to represent themselves as older avatars in virtual environments. To understand the needs and preferences of older adults in relation to representational avatars, we conducted two workshops in which 25 older adults (aged 70+) designed a humanoid virtual avatar using an open source avatar creation tool. This study aimed to explore how older adults would choose to represent themselves as a humanoid avatar. Our findings underscore the diverse views of older adults in relation to self-representation. Participants created avatars that ranged from Actual Avatars (realistic representations of age), Vibrant Avatars (idealised representations of self), 'Other' Avatars (representations of self as another person), and Companion Avatars (the creation of a persona that would act as a companion). Through this process, our participants negotiated stereotypes of ageing, either by choosing to create realistic representations (thereby rejecting societal ideals of youth), or by carefully crafting vibrant versions of themselves or others. Our findings extend current conceptualisations of avatars and show that avatar creation tools need to be designed to allow the representation of the full range of human characteristics, including those associated with age. 


\section{PRIOR WORK}

\subsection{Older Adults Negotiating Stereotypes}

The activities that older adults do on Information Communication Technologies (ICTs) may question ageist stereotypes. Stereotypes are simplified beliefs that depict a set of attributes to all persons in a category [18]. In the case of older adults and their relation to ICTs, there are social assumptions that older people are incapable of using technologies [10]. In contrast to these beliefs, older adults are using ICTs to communicate through email, play videogames [17], do travel reservations, do shopping and in less percentage to participate in groups with shared interests [32]. Older adults who are using these technologies have overcome ageist stereotypes suggesting new ways of involvement.

Older adults do not replicate the interests and behaviors of younger people. While online social networks may have characteristics that can motivate social participation, the lower percentages of older people using these technologies show that they may not adjust to older adults' preferences [7]. On the other hand, the popularity of online communities (for example SeniorNet, Silver Surfers) targeted specifically for seniors is increasing and may have more suitable features for this population. Research should focus on what is attractive for older adults in these environments. A study that explored what participants value on GreyPath.com, one of Australia's largest online communities for seniors, conclude that they value to be part of a "community of peers" [5]. While having access to online communities of people who are living similar situations may generate empathy [5], other interventions showed that the preferences of participants in this medium varied according to the group of people they want to communicate [19]. In addition, a study that used a prototype of an application to share pictures and texts within a group of older people found that this was also a way to provide opportunities of self-expression and creativity [37]. All these examples suggest that older people are interested and capable of using information communication technologies, but they may also be looking for specific uses that respond to their requirements.

If older adults have diverse preferences regarding the use of ICT, it is worth exploring their inclinations regarding their online visual depiction. As virtual environments become more complex, we need to interrogate more sophisticated ways of selfrepresentations.

\subsection{Avatars}

An avatar is a representation of the person that exists in a virtual environment (VE) (typically in an online game) and is most commonly represented graphically. An avatar does not need to accurately represent the users' physical traits. In fact, they can serve as "tangible embodiment" of the user's identities [7:1151]. Furthermore, Turkle [35], noted that through these avatars, VEs offered opportunities to project conscious and unconscious aspects of the self, suggesting that VEs serve as places to work through unresolved issues. Even though the industry trends are typically focused on achieving photorealism, studies have shown that avatars do not need to be humanoid, photorealistic or look like the user when representing the user's identity [8]. Still, humanoid avatars are recommended for fostering social interactions, because they incorporate human characteristics that are familiar to real life [42]. Users explore these interactions through different types of avatars.

Researchers have defined different classifications of avatars in VEs in the literature. Turkle's ethnographic studies proposed two main types of virtual identities: the Ego Ideals who explore ways to achieve the ideal selves and the Role Players that are constantly exploring something different from the real self [35]. Turkle states that Role Players question the nature of identity as they provide unlimited self-representations and the "possibilities to be many" [35:164]. Neustaedter and Fedorovskaya [26], identified a category called the Realistics, a third differentiation based on how users designed avatar representations but also developed avatar identities through VEs. Realistics referred to persons who tried to replicate their actual appearance and personality in the virtual world overlapping the activities of the VE with their real life [26]. In order to project these identities, some VEs allow users to manipulate their identities through customization systems.

Players often spend a significant amount of time customizing their avatars to reflect their personality or to project imaginary identities [41]. In fact, greater similarity between the avatar and the user's appearance or desires is a predictor of major autonomy, immersion, devoted effort, enjoyment and positive affect [3]. In fact, Yee states that virtual avatars can influence the way users think and behave in the VE [41]. For example avatars that depict similar visual characteristics to the user have been shown to be effective in promoting real world health behavior [1]. Thus, avatars do not only serve representational purposes, their creation and customisation also contributes to ongoing engagement.

Although customization systems offer great range of options when personalizing an avatar, the interface's affordances influence the avatar creation process. When users start building their avatars they do not always have a clear visual model of what they are making. Therefore, the choices of the interface guide the user in the creation of their depiction. As researchers point out, an avatar is "the result of a dialogue between user and interface" [20:239]. McDonough stresses that because the designers of virtual worlds used to be young middle-class white males, the resulting VEs were limited in their conceptions of an avatar [21]. However, these designers may not fully understand how different groups of users want to represent themselves online, especially when there are new user groups venturing in to VEs. Older adults are a new user group in virtual environments and online games, but little is known about how they want to represent themselves as virtual avatars. It is important to consider older adults' requirements to influence the design of avatar customization systems.

\subsection{Older Adults and Avatars}

Older adult populations are increasingly using virtual environments (VEs) to keep the mind active, have fun, maintain 
social connections and improve physical fitness [4]. Almost half (43\%) of Australians over 65 years of old play online games, representing the fastest growing group of users in this market. Additionally, older players' populations will continue to rise as baby boomers age. Moreover, even younger players, consider online games as a tool to promote ageing well [4]. Thus, the continuous growth of players over 65 years old requires the attention of developers and researchers.

VE preferences among older adults are characterized by diversity, but there is an emerging interest toward the use of avatars. De Schutter [29], state that older adults prefer simple online games familiar to physical games that they had previously played. In contrast, Pearce, revealed that baby boomers favored games of roleplaying or adventure, VEs with rich narratives that provide an "escape to another world" [27: 153]. Avatars are key components of these types of games.

Avatars can allow older adults to experience a new body representation that can facilitate social interactions and enjoyment of virtual physical activities. Research by Siriaraya et al. [31], indicated that older adults in a social VE use ageless avatars to blend in younger populations by anonymising their ageing characteristics. In their study, older adults with physical disabilities projected virtual bodies that did not mirror their body limitations, giving them the opportunity to enjoy bodily activities such as dancing without the requirement of their physical movement. Using avatars also allowed older adults to bring expertise from the physical world into productive activities in VEs, such as teaching how to train dogs [31]. Finally, avatars in this study facilitated social engagement by visually expressing emotions. The social capacity of avatars was also depicted in a study of older players of World of Warcraft who used the game to maintain relationships with family and friends, but also as a space to build meaningful new friendships [43]. These possibilities to increase social interactions are important given the strong initiatives to promote social inclusion among older adults [40].

Given that older adults are increasingly using VEs and that even players of younger ages consider online games as a tool for aging well [4], it is important to give attention to how older people are represented in these spaces. The creation of an avatar is usually done at the beginning of an experience in an online game. However, there is very little research exploring the use of avatars by older adults and how they can provide opportunities for exploring identities. As we have mentioned, studies suggest that older adults create ageless avatars $[8,28,31]$, but it is not clear if this is the purpose of the users or a limitation of the interface which may not be providing choices to make their avatars look more like themselves. Our study aims to understand this issue by exploring representational requirements of older adults designing a humanoid avatar.

\section{METHODS: OLDER ADULTS AS AVATAR DESIGNERS}

This research used a human-centered exploratory approach [25] in which older adults created their avatars in a group workshop setting. The focus aligns with HCI literature that suggests that older adults' voices should be central in research involving the design of technologies for later life [36].

We ran two avatar creation workshops, one in a rural and one in an urban setting with a total of 23 participants aged between 70 and 80 years old. These workshops informed subsequent sessions among a larger series of workshops exploring the use of avatars and virtual reality technologies to facilitate social participation among older adults. The sessions were held in computer laboratories at the university where each participant had access to a personal computer with Makehuman software. This is an open source 3D graphic software that is designed for modelling virtual humans. Users start with a standard androgynous human form that can be modified with great detail through different body parameters (such as height, weight) or muscularity among others. The software uses a simple graphical user interface with sliders to manipulate the tridimensional shapes (Figure 1).

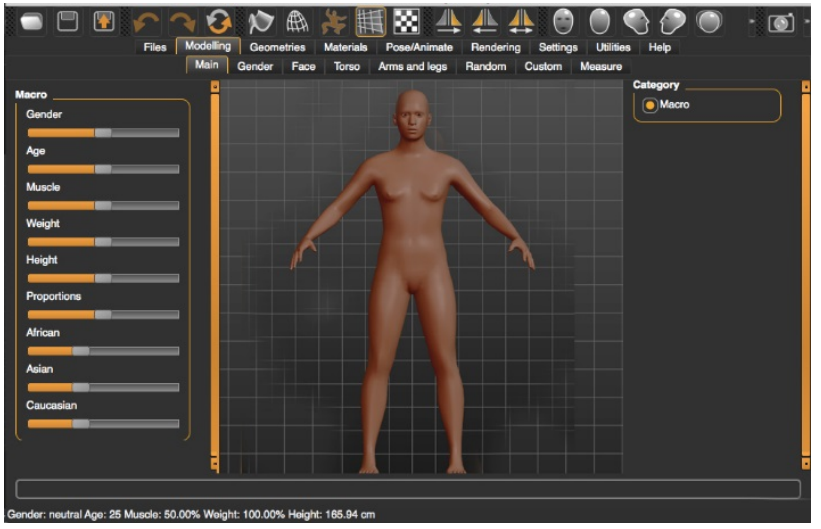

Figure 1: MakeHuman interface showing the initial androgynous human form

\subsection{Participants}

A total of 23 older adults, 14 males and 9 females between 70 to 80 years old participated in the study (Table 1). The study was conducted in both a rural and an urban setting of Australia with two circles of 'Technology Explorers', groups of older adults who meet monthly to explore virtual reality technologies. Older adults were recruited using purposive sampling techniques. Strategies included both online and offline methods, such as advertising in newsletters, through the project website and in community centres and local libraries. Participants had reasonable experience using smartphones, tablets and desktop computers. However, almost no participants had experienced virtual reality or online games and nobody had previously created an avatar. The Research Ethics office of our university approved this study (\#1647456) and participants provided informed consent.

\subsection{Procedure}

The workshops were run during a 90 -minute session. This was sufficient time for participants to explore the main features of the interface, to create an avatar, and to discuss the experience. The 
participants were guided to create an avatar character that they will use in VEs to interact in future workshops with other participants of the group. The facilitator guided the exploration of the software through a projection that illustrated his instructions and served as a visual support that all participants were able to see. Additionally, during the whole period, there were helpers assisting participants' use of the software.

Through this process, participants firstly customized the avatar's general parameters as gender, age, body shape and ethnicity through an African, Asian and Caucasian slider. The age slider ranges from 1 to 90 years old and affects the shape of the body, generates wrinkles and modifies the posture. Participants then configured specific parts of the body, the skin and the clothes. The skin tab offered textures according to gender, ethnicity and age stages (young, middle age and old). The old skin added age lines to the texture of the body. At different stages of the workshop, participants were instructed to record screenshots of their avatars. Following the session, we photographed participants in order to compare their physical traits with their avatar's parameters. Finally, the participants discussed their experience of creating an avatar through informal conversations over a shared lunch.

After the workshop, we emailed participants a screenshot of their avatar and asked them to complete an online open-ended questionnaire about the avatar workshop. Of the 23 participants, 20 completed the questionnaire. Three participants did not provide responses to the questionnaire, which was completed in their own time after the workshop had ended. It is possible these participants did not have time to complete the questionnaire, but we do not know why exactly they chose not to respond.

\subsection{Data Analysis}

The research data consisted of: audio recordings of conversations between participants and researchers during the workshop; field notes from researchers who observed the workshops; screenshots of participants' avatars captured at various stages; worksheet with avatar characteristics; photographs of participants; and questionnaire responses. The data were imported into NVivo. We replaced participant's names with pseudonyms, which were selected by participants (for all except three cases). We excluded the data of one participant due to a technical fault in the design of the avatar. We organized the data into individual profiles as we were comparing avatars with participants. Then, we coded the materials iteratively using a thematic analysis relevant to the research questions [25].

\section{RESULTS}

Participants stated that creating an avatar was a fulfilling experience. In relation to the goal of the workshop, some participants argued that having a firmer context of use for the avatar would have better clarified their design, whereas others appreciated that the workshop did not point to a specific goal as they felt free to make their own choices. Having analysed the data, participants' avatar designs fell into four categories (see Table 1) that built of previous literature and in which the findings are structured.

Table 1: Socio-demographics and avatar's names of the participants categorized according to their avatar's types

\begin{tabular}{|c|c|c|c|c|}
\hline $\begin{array}{c}\text { Avatar } \\
\text { Category }\end{array}$ & $\begin{array}{c}\text { Pseudony } \\
\text { m }\end{array}$ & Age & G & $\begin{array}{c}\text { Avatar's } \\
\text { Name }\end{array}$ \\
\hline \multirow{7}{*}{$\begin{array}{l}\text { The Actual } \\
\text { Avatar }\end{array}$} & Bob & 74 & M & Fred \\
\hline & Gordon & 78 & M & Tezza \\
\hline & GT & 70 & M & GT \\
\hline & Keki & 80 & M & $\mathrm{KP}$ \\
\hline & KennyG & 73 & M & KennyG \\
\hline & Tony & 74 & M & ALikelyLad \\
\hline & TR & 73 & M & James \\
\hline \multirow{8}{*}{$\begin{array}{l}\text { The Vibrant } \\
\text { Avatar }\end{array}$} & Amy May & 72 & $\mathrm{~F}$ & Alex \\
\hline & Forjador & 70 & M & Forjador \\
\hline & Herbert & 71 & M & Kevin \\
\hline & Nik & 79 & $\mathrm{~F}$ & Nik \\
\hline & Sally & 70 & F & Ruth \\
\hline & Tom & 74 & M & Jungenrog \\
\hline & Violet & 77 & $\mathrm{~F}$ & Bluelyn \\
\hline & Yulia & 74 & F & Anastasia \\
\hline \multirow{5}{*}{$\begin{array}{l}\text { The Other } \\
\text { Avatar }\end{array}$} & Herb & 73 & M & John \\
\hline & Klaus & 79 & M & Don Fulano \\
\hline & Mandy & 76 & $\mathrm{~F}$ & Jayda \\
\hline & Red Mog & 79 & M & Thomas \\
\hline & Mariam & 76 & $\mathrm{~F}$ & Mariam \\
\hline \multirow{2}{*}{$\begin{array}{l}\text { The } \\
\text { Companion } \\
\text { Avatar }\end{array}$} & Bernard & 77 & M & Anthea \\
\hline & Cleo & 75 & F & Steve \\
\hline $\begin{array}{l}\text { Not in Data } \\
\text { Analysis }\end{array}$ & Holly & 80 & $\mathrm{~F}$ & Hollypolly \\
\hline
\end{tabular}

\subsection{The Actual Avatars}

This group resonates with Realistics avatars that replicate real life physical representations of the users [26]. The participants who created Actual Avatars focused on corporeal traits to make their avatars look older. Some of the ageing characteristics depicted by the Actual Avatars are wrinkles, less hair in head, reduced muscular tone and alteration of the stance.

Seven male participants created an avatar depicting their actual selves (see Table 1). In fact, it was a clear goal for some of them, as described by Gordon (male, 78): "I tried within the time available to create a replica of me", commenting: "When I was 50, I wanted to be 25, but now ... that I am almost 80. You say: why should I try to do things as a 40 year old? I'm used to 
being, and I'm happy, you know, I'm skinny... I like to be more muscular, but I don't have to be, I can do things as I am ... I can be myself... and go skydiving; I don't have to be a 25-year-old person".

Tony mentioned that he preferred to begin with an avatar that looked like him because he did not yet know the context in which it would be used: "I don't know what scenarios we are going to do. I'm me for now" (Tony, male, 74). Tony may be implying that he will do more exploration of avatar identities after having a better understanding of the contexts. However, not all the participants had a clear idea of why they created an avatar that resembled their actual self, as noted by GT (male, 70) who mentioned, "I went with the flow".

When creating the Actual Avatars, participants chose parameters similar to their physical characteristics. For example, Gordon's avatar tries to depict his real physical traits: male gender, $180 \mathrm{~cm}$ of height, blue green eye colour, Caucasian ethnicity, short hairstyle, 80 years of age, and an 'old skin' texture. Gordon spent considerable time designing his facial features reflecting that it would be useful to have a mirror to recreate his face more accurately (see Figure 2).

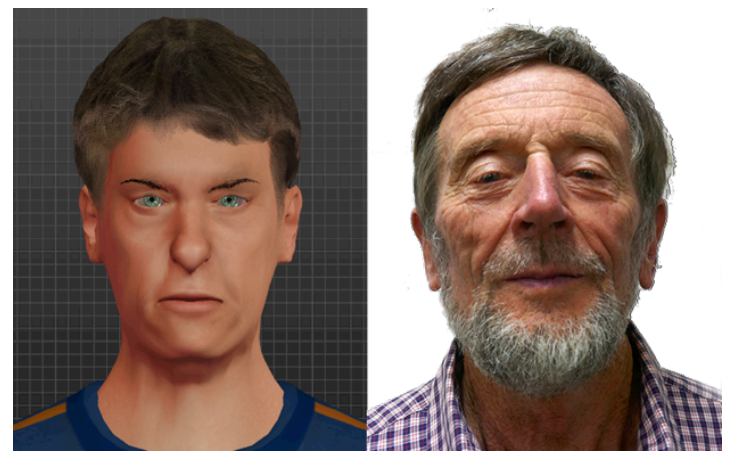

Figure 2: Gordon's avatar depicting his Actual Avatar

Other participants gave more importance to the body shape. For example, Keki modified the depth and position of the avatar torso and hip depicting some similarity with his body shape (see (see Figure 3). Tony and TR added a low level of 'pregnancy stomach' trying to simulate their body figure (see Figure 4). After creating the avatars, some of the participants criticized the software stating that the age control was not faithful and that their avatars looked younger than the age they had inserted.

Additionally, participants mentioned that they would like to add a beard and have less hair on the head. KennyG (male, 73) was worried about the tight stance of the avatar, mentioning that he had never walked like that. Tony and GT were interested in having more facial expressions and Tony specifically mentioned that he would like to "change its [the avatars] unsmiling mouth." Finally, Gordon stressed that creating an avatar that resembles the user is more difficult than making up an avatar and indicated that he would need more time to learn how to use the tool and configure all the settings in a more desirable way.

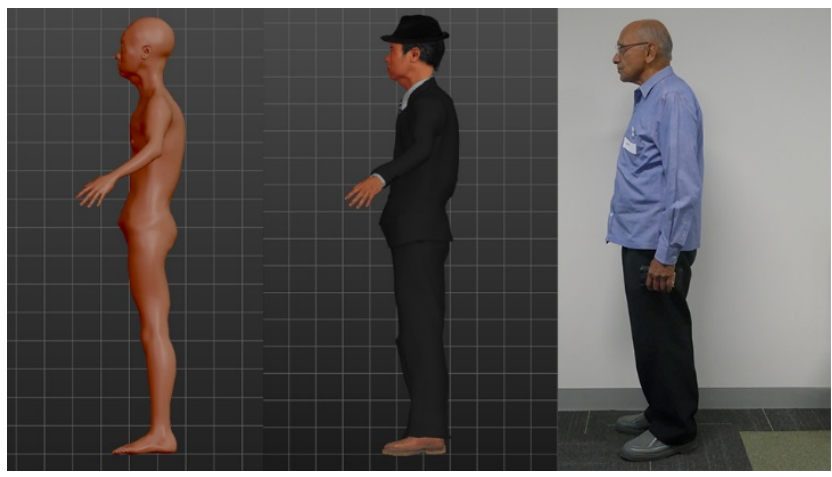

Figure 3: Keki's avatar with the torso and hip manipulation

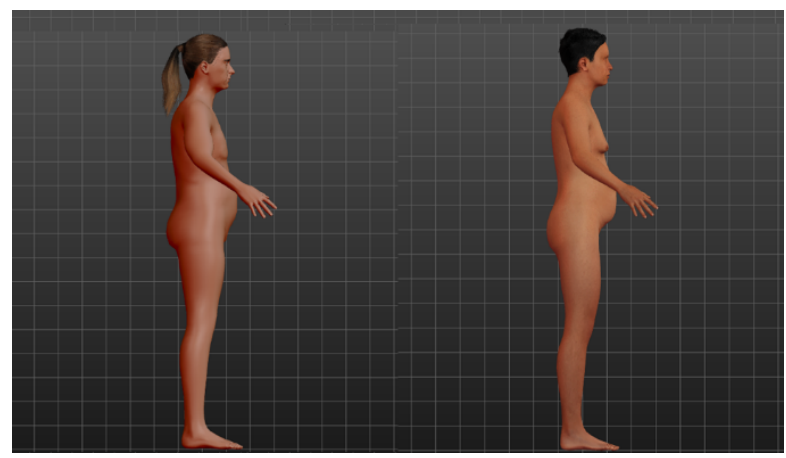

Figure 4: Tony's (left) and TR's (right) avatars illustrating a low level of 'pregnancy stomach'

\subsection{The Vibrant Avatar}

This category builds on Turkle's Ego Ideals [35], who alleges that users recreate an avatar enhancing their physical traits. Even though participants in this category were following Western cultural ideals of beauty, it seems that it was more important to enhance the physical condition of the body over the aesthetical appearance of the avatar. Vibrant Avatars prioritized the physical condition, augmenting traits to look healthier, stronger and younger. Eight older adults created Vibrant Avatars: three added these features to an actual version of themselves, while five created younger selves.

\subsubsection{The Vibrant Elderly Avatars}

These participants depicted avatars with truthful physical traits such as age, height, ethnicity and 'old Caucasian skin' (see Figure 5). Although the avatar designed by Herbert (male, 71) reproduced many of his features, he mentioned that he was creating an idealized version of how he would like to be 'now', with 'height', 'shoulder width' and 'strength'. Likewise, Sally (female, 70) stated that she was creating an avatar of someone looking like she "would want to", a "healthy looking" avatar with "good physique" to which she would like to add more age lines. Throughout the workshop, she stated that she wanted to depict a strong female body adding that the possibilities of clothes that make a woman 
look "strong" were limited. Analysis of her avatar suggests that she may have tried to achieve this by increasing muscle mass and choosing a male outfit. Additionally, at the end of the session and referring to Makehuman software, Sally expressed that "setting characteristics is 'lumpy". Similarly, Nik (female, 79) was concerned that she could not find suitable clothing for her avatar stating "I'd rather have some of the male clothes on her. There's not a lot of female in her!". While depicting a 78-year old avatar, Nik configured a tall female character $(238 \mathrm{~cm})$ with high muscle mass (85\%), and large firm breasts. She selected a ponytail similar to the one she was wearing the day of the workshop and noted that she was happy that her avatar has no necklines (see Figure 6).

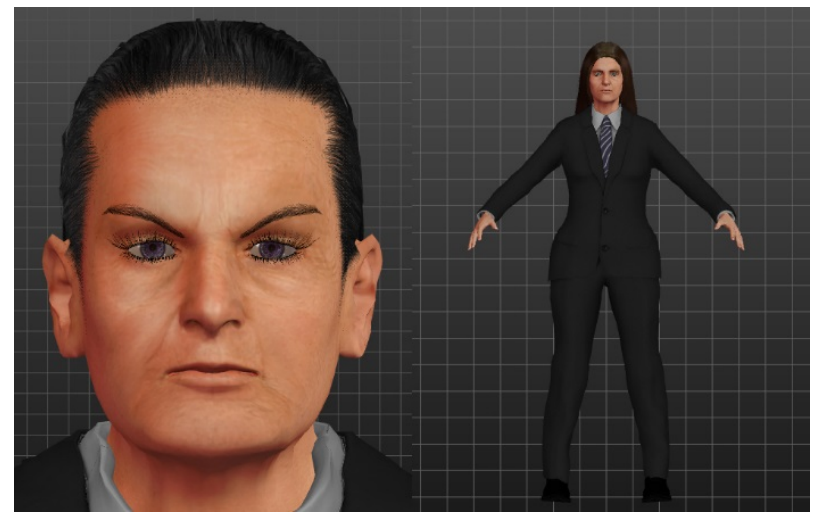

Figure 5: The Vibrant Elderly Avatars: Herbert's avatar (left) and Sally's avatar depicting a "strong" woman (right)

\subsubsection{The Vibrant Young Avatar}

The five participants in this category created younger versions of themselves while emphasizing traits that made them look vibrant. To illustrate this, Forjador (male, 70) created an avatar with his "state of fitness at 30 years old" and Yulia (female, 74) created a 40year-old avatar (see Figure 6) saying "if I was going to create an avatar, I was going to create somebody with strength, bodily strength and few brains... You know somebody that was able to do, abled body, able to do whatever I wanted to do." A factor that may have influenced Yulia's wish to create a strong character was that on the day of the workshop, she forgot to take her pills and was experiencing weakness and difficulty walking, often excusing herself for being slow. Another participant who reflected on her body was Violet (female, 77) who created a 30-year-old version of herself mentioning that her avatar "... stands better than what I do". These comments suggest that participants wanted to depict the strength their bodies are missing.

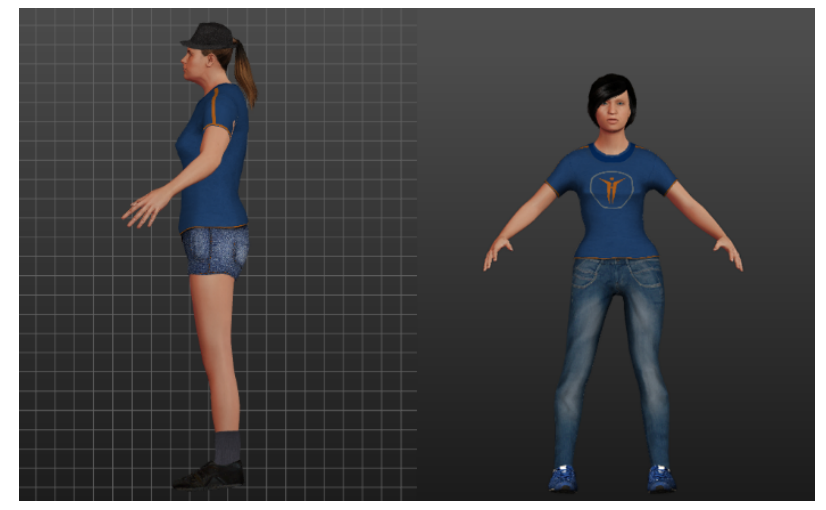

Figure 6: Nik's Vibrant Avatar (left) depicting her ponytail and idealized traits and Yulia's avatar (right) with strength

Amy May (female, 72) was also looking to depict strength in her 35-year old avatar but additionally she was exploring a taller representation of herself by making her avatar grow from her actual height of $158 \mathrm{~cm}$ to $173 \mathrm{~cm}$. Amy May was designing an "androgynous" avatar, "not particularly curvy..." as she doesn't believe looks are everything. She named her avatar 'Alex' so it can be genderless. Amy May was concerned by the high level of breast configuration available in the interface insinuating that a man designed the software.

Tom (male, 74) reflected on the creation of a younger muscular avatar revealing that if he had the possibility to re-run his life, he would prefer to have the experience of a 70-year-old person than the strong body of a "sort of superman at 25" (see Figure 7).

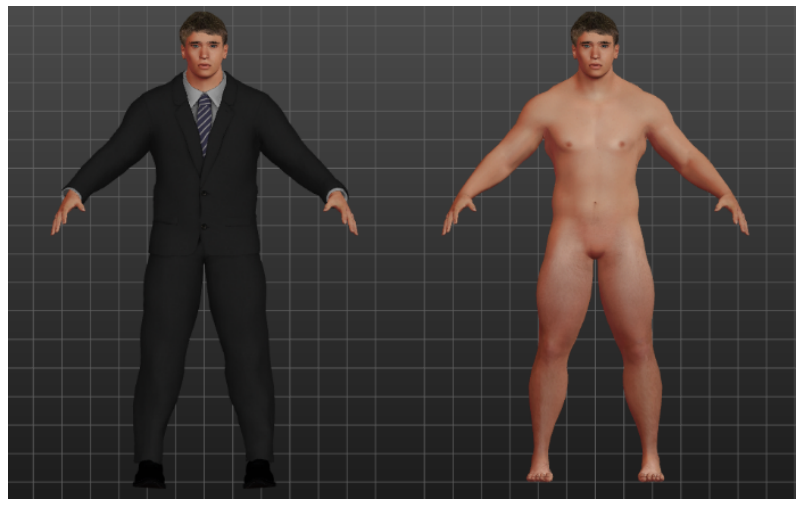

Figure 7: Tom's avatar depicting his “superman" body

These participants created their avatars reflecting on what they want to project to others. For example, Forjador (male, 70) said, "... if I am interacting with other people, I don't want to be feeble old man or a fat slumber." He added that his avatar has a good age (30 years old) for socializing. Tom (male, 74) also talked in relation to the social setting stating: "I felt if it was to represent me interacting I wanted it to be as much like my features as possible but I also wanted its physical characteristics to be capable of 
change." This may suggest that he wanted to adapt his avatar in relation to the environment while still being recognizable.

The names of the participants depicting the Vibrant Avatars were more ludic than the Actual Avatars showing that they were more open for exploration and not so faithful to reality. Forjador named his avatar with a name he had to invent when attending a writing class in high school. Tom called his avatar Jungenrog alluding to a younger version of himself and Nik used her nickname from her “...long lost youth!!!”. Violet named her avatar adding the color of the avatar's clothing to her name: Blue[name]. Finally, Amy May named her avatar Alex so it can have any gender.

\subsection{The Other Avatar}

The Other Avatar resonates with Turkle's Role Players [35], who explore other identities through the visual representations of avatars. Five participants created personas different from their actual selves. For example, Herb (male, 73) created a character depicting "brutal physique, cold eyes, having great presence, thick set neck" (see Figure 8). Reflecting on his avatar Herb said "I have always been such a timid, little person, to have somebody who is bold and strong I can re live something entirely different here... My other person could come out bit further". Herb's avatar, John is named after the song Big Bad fohn by Jimmy Dean (1961) [38]. The song is about a strong miner who sacrifices his life to save everyone after a mine collapses. In the case of Herb his avatar has the physical features of this character but in contrast to its ferocious look, the song tells a story of courage and sacrifice. This illustrated that the names of the avatars in this category play an important role depicting a background and personality of other persons.

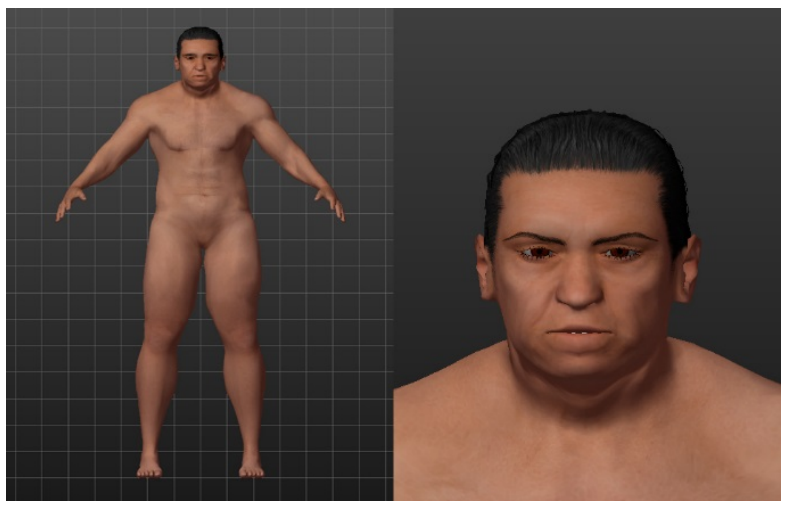

Figure 8: Herb's avatar illustrating his "brutal physic"

Mariam (female, 76) also liked to have an avatar different from her appearance. She created a $100 \%$ Asian character, contrasting her Caucasian ethnicity and expressing that it was a version of the other side of her family, who were Malaysian. In fact, the avatar was named after her niece.

Another example of this category is Klaus's (male, 79) neutral avatar, which is not representing his physical image, but an average person (30\% Asian, African and Caucasian). He named his avatar Don Fulano, an Spanish expression to refer to "an indeterminate or imaginary person" [12]. Klaus mentioned that he liked the ordinariness of his avatar.

Two participants designed and named their avatars to represent their grandchildren. Mandy (female, 76) recreated her 15 -year-old granddaughter and Red Dog (male, 79) his 28-year-old grandson. Red Dog mentioned that he wanted "a young, smart man who could converse on almost any subject" stating that it was also important that he was tall, "well built" and had eye colour he could "trust". It seems that participants were not looking to recreate their grandchildren, but to represent themselves in the image of their grandchildren.

\subsection{The Companion Avatar}

The Companion Avatar refers to constructions of identities inspired to bring company and not to represent the user. Two participants created other personas as companions. Bernard (male, 77) created a 70-year-old female travel companion named after a friend he knew 50 years ago (see Figure 9). When explaining why he created this avatar, he said "... a friend cos I've seen enough of myself. I'd like someone else." Afterwards, he mentioned that he would like to make the avatar look happy, as "currently she has a sour look."

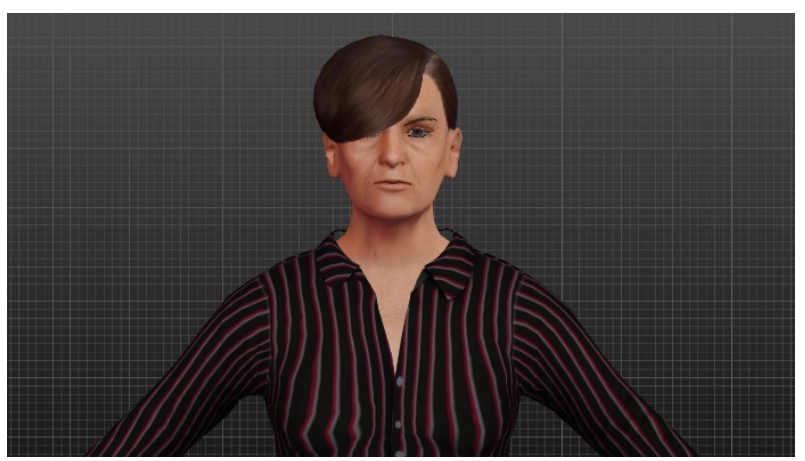

Figure 9: Bernard's avatar: a female travel companion

Cleo (female, 75) created an avatar of her deceased husband to see if she can "connect" with him through the VE. These two participants created avatars inspired by real persons they met in their past, which may indicate that they would like to maintain those relationships.

\section{DISCUSSION}

In this study we aimed to understand the representational requirements of older adults creating virtual humanoid avatars. The process resulted in a negotiation of visual features influenced by desires of the user, societal norms and limitations of the interface. Designing an avatar was reported as being an enjoyable experience as it allowed older adults to explore the complexity of 
identities in later life. We build on three categories of avatars that have been previously identified in the literature and propose a fourth category: the Companion Avatar recognized in this study. We will discuss how older adults in this study depict specific visual requirements concerning their virtual representations.

\subsection{Actual Avatars: Acceptance of Ageing Bodies}

Creating a lifelike avatar among older adults may have greater significance than just reproducing the actual appearance of the self into the depiction of an avatar. Constructing an Actual Avatar may suggest that participants are reaffirming their ageing body representation, and are not trying to look younger or follow social stereotypes. Thus, they incorporate older adult features in their avatars without considering other possibilities. These participants may not be linking the Actual Avatar with negative stereotypes of later life as suggested by Gordon who perceives that the Actual Avatar won't limit how he uses the VE.

As indicated previously, only male participants created Actual Avatars depicting that women in this study may be not willing to portray their ageing bodies through the avatars. This may be because women in society gain status in relation to their appearance [39] which is judged by beauty ideals that generate body dissatisfaction in most of women across the life span [33]. Even though previous research suggests that women become less concerned about their appearance when they are quite elderly [33], our study insinuates that our female participants preferred to project other depictions than the ageing bodies onto their virtual avatars. In contrast, men in society gain status through a broader set of qualities such as wealth, intelligence and power [39] and therefore may be not so concerned by physical appearance. Moreover, studies suggest that age-related appearance changes in men are viewed relatively neutrally or even as a way of enhancing male attributes [15].

The requirement of some of our participants to depict avatars with ageing bodies contradicts previous studies that had suggested that older users prefer to create avatars different from their real bodies, and making them conform with Western ideals of beauty [9]. In this study, some older adults created actual depictions of themselves. Others idealized their representations as we describe in the next section.

\subsection{The Vibrant Avatars: Overriding Ageing Stereotypes}

Participants in the Vibrant Avatar category idealized the physical condition of the virtual avatars, prioritizing traits that made their virtual bodies look healthier, stronger and younger. A possible explanation of this preference is that health and physical abilities largely mediate body image satisfaction among older adults [2]. Yulia illustrates this point clearly when trying to create an 'abled body' who prioritised strength over brains. It is remarkable that the characteristics of a better physical condition are not easy to visually depict primarily for female avatars who aim to maintain feminine attributes. Female participants were exploring diverse options such as adding muscles, modifying the stance, the age or even adopting clothes to make them look stronger, active and healthy. One of the customization options that participants used in this category was the configuration of bodies with younger ages. As other researchers affirm, older adults who feel younger than their chronological age preserve a positive image of themselves [16]. It is possible that creating an avatar with a younger representation might bring similar consequences in older adults. Investigators have suggested that avatars can make users change the way they think and behave [41]. In addition, the fact that older adults build younger virtual bodies is in accord with previous research that suggested that younger avatars could provide anonymity to older adults with opportunities of blending with younger populations in virtual worlds [31].

While some participants in this category augmented their actual ageing bodies with vibrant traits, others not only amplified their bodies but also hid some physical traits such as body shape and frailty. Avatars can be used to go beyond the specific body appearance to cover traits that Western cultures often view in negative terms [26]. However, in contrast to previous studies with younger populations who were principally idealizing their avatars to imitate Western standards of beauty $[9,26]$, older adults in this study gave more importance to physical condition. This conforms to the view that older adults shift their concerns from physical appearance to physical condition [2]. This may also explain why older adults were focused on creating Vibrant Avatars over 'beauty' ones. Analyzing this in the case of women shows how they are also negotiating gender issues by giving less importance to physical appearance. Nevertheless, this does not mean that physical appearance is not important in later life as was shown by participant's comments about the limited hairstyles and clothing available in Makehuman. It merely suggests that it is not the central focus in their lives.

Some older adults stated that they designed their avatars in relation to a social context. This finding aligns with previous studies that suggest that users make decisions about their avatar's appearance to make an impression to others [11]. Older adults in this category might have been negotiating with vibrant features to project an idealized version of themselves into social contexts. However, the association between avatar representations and social contexts should be explored with more detail because in this study we did not provide a precise specification of a VE, game or context of use.

These idealized depictions of those in the Vibrant Avatar category are suggestive of attempts to override negative depictions of older adults such as frail, dependent and powerless [14]. Moreover, considering that individuals might internalize negative perceptions affecting their own conception in a pessimistic manner [14]; the Vibrant Avatar might support more optimistic self-perceptions of older people. Further study should analyze not only the creation but also the use of avatars in VEs among older adults. This may provide more understanding if the characteristics of the avatar depictions influence the type of experiences chosen by older adults, for example identify if Vibrant Avatars empower people to explore activities that require more physical condition such as climbing. 


\subsection{The Other Avatar: being other personas}

The Other Avatar category shares similarities with the Role Players type detailed by Turkle [35] in that it allows users to explore other personas. Older adults share the interests of younger populations of exploring identities different to their self-representation. Many of these characters are imbued with deeper meanings, such as Herb's avatar John, inspired by the song Big bad fohn or Klaus's avatar Don Fulano (Spanish version of Joe Blow). One of the explanations of creating Other Avatars is that they may confer anonymity, which has been previously attributed to this category[13, 35].

However, one of the subgroups of this category that may be unique to older adults is the depiction of grandchildren. Other researchers postulate that grandchildren represent an extension of older adults' self beyond their lifetime [34]. Grandchildren's bodies may be portraying some of the traits of their grandparents in a fresher and vigorous way. Therefore, it is probable that older adults in this study, created these avatars as a means of expressing an extended version of themselves.

\subsection{The Companions: bringing nostalgic relationships}

The creation of Companion Avatars by two of our participants might also be a result of experiencing the social consequences of ageing. As older adults age the opportunities for socializing diminish [40]. Therefore is it through virtual reality that Bernard may be finding a way to create idealized female friend with whom he can share his interests. He named his avatar after a person he knew fifty years ago which may be suggesting that he would like to continue this relationship. In the case of Cleo, she depicted her deceased husband commenting: "I wanted to see if I can connect back to him". Interestingly, these two participants based their avatars on real people from their past. Previous experiences are an important part of a person's identity [30] and in this case, persons from the past might be linked to previous roles that were a significant part of older adults' identity. For example, Cleo is depicting her deceased husband, which may be a way to reinforce her identity as a spouse. It is believed that through these roles people feel a sense of belonging in a group [6]. In later ages, these roles frequently become less clear as the circumstances change [40]. Avatars may provide the opportunity to bring companions to life, but also allow participants to revisit the roles that are linked to them.

We believe that the Companion Avatar is a new category of depiction when representing the user. It may have a similar function to autonomous virtual agents that provide company [23]. However, these agents have a separate identity that does not represent the user, whereas companions in this study were intended to be their self-representations.

\subsection{Constraints of the interface}

Even though Makehuman offers a great amount of configuration in relation to the body shape, a majority of participants mentioned the software limited the customization of their avatars. Female participants argued that the software did not offer enough female options of clothes and hairstyles, while it gave an exaggerated configuration for some female traits, such as breasts. These comments further highlight concerns about gender depictions that have previously been highlighted in the literature [21]. Regarding those who were portraying avatars that looked older, they aimed to have more control of ageing traits, such as age lines, amount of hair, body posture and clothes. Additionally, many participants mentioned that they would like their avatar to have a more natural expression. This might be related to the 'Uncanny Valley', a perception that the level of human realism in avatars is not perfectly accomplished, affecting negatively the user experience [24]. Finally, participants highlighted this concern focusing on the expression of more positive emotions as a "happy look" which might be additionally depicting their intentions to create positive self-representations. These requirements are informing the configuration of an avatar customization system that will be evaluated with the same group of participants in a subsequent workshop. This will deepen our understanding of avatar customization preferences among older adults.

\section{CONCLUSIONS}

In this paper we have presented the findings of 23 older adults above 70 years old who created humanoid virtual avatars with Makehuman. The experience of creating a virtual character depicted specific representational requirements of older adults when creating a humanoid avatar. We identified four categories of avatars: the Actual Avatar, the Vibrant Avatar, the Other Avatar and the Companion Avatar. These avatars are a consequence of older adults' preferences illustrating for some the acceptance of their ageing body, the desire of an augmented physical condition, the wish to embody other personas and finally the necessity to bring people as a company. Among all these categories the negotiation of societal ideals of youth is concurrent and while some participants demanded the faithful representation of ageing features such as age lines, others were exploring mechanisms to enhance vibrant versions of themselves or to recreate others. We built in three previously known categories of avatars, and propose the Company Avatar that to our best knowledge, is a new category of avatar.

Our study depicted that avatar customization systems should incorporate the representation of a wider set of humanoid features as those that visually represent age.

\section{ACKNOWLEDGMENTS}

This research is supported by the Australian Research Council and the Microsoft Centre for SocialNUI. We thank Thuong Hoang and Jeni Warburton for their invaluable support at different stages of research.

\section{REFERENCES}

[1] Ahn, S.J. 2015. Using avatars and agents to promote real world health behavior changes. The Digital Patient: Advancing Healthcare, Research, and Education. and C.M.B. C. Donald Combs, John A. Sokolowski, ed. John Wiley \& Sons, Inc. Published. 171-180. 
[2] Baker, L. and Gringart, E. 2009. Body image and self-esteem in older adulthood. Ageing and Society. 29, 6 (2009), 977-995. DOI:https://doi.org/10.1017/S0144686X09008721.

[3] Birk, M. V, Atkins, C., Bowey, J.T. and Mandryk, R.L. 2016. Fostering Intrinsic Motivation through Avatar Identification in Digital Games. Proceedings of the 2016 CHI Conference on Human Factors in Computing Systems - CHI '16 (2016), 2982-2995.

[4] Brand, J.E., Todhunter, S. and Jervis, J. 2017. Digital Australia 2018.

[5] Burmeister, O.K. 2012. What Seniors Value About Online Community. The Fournal of Community Informatics. 8, 1 (2012), 1-12.

[6] Carroll, J.M., Hoffman, B., Han, K. and Rosson, M.B. 2015. Reviving community networks: hyperlocality and suprathresholding in Web 2.0 designs. Personal and Ubiquitous Computing. 19, 2 (2015), 477-491. DOI:https://doi.org/10.1007/s00779-014-0831-y.

[7] Charness, N. and Boot, W.R. 2016. Technology, Gaming, and Social Networking. Handbook of the Psychology of Aging. Elsevier Inc. 389-407.

[8] Cheong, W.L., Jung, Y. and Theng, Y.-L. 2011. Avatar: A Virtual Face for the Elderly. Proceedings of the 10th International Conference on Virtual Reality Continuum and Its Applications in Industry - VRCAI '11. (2011), 491-498. DOI:https://doi.org/10.1145/2087756.2087850.

[9] Ducheneaut, N., Wen, M.-H., Yee, N. and Wadley, G. 2009. Body and mind. Proceedings of the 27th international conference on Human factors in computing systems - CHI 09. (2009), 1151. DOI:https://doi.org/10.1145/1518701.1518877.

[10] Durick, J., Robertson, T., Brereton, M., Vetere, F. and Nansen, B. 2013. Dispelling Ageing Myths in Technology Design. Proc. OzCHI 2013 (2013).

[11] Fong, K. and Mar, R.A. 2015. What Does My Avatar Say About Me? Inferring Personality From Avatars. Personality and Social Psychology Bulletin. 41, 2 (2015), 237-249. DOI:https://doi.org/10.1177/0146167214562761.

[12] "fulano," R.A.E. 2014. Diccionario de la lengua española [Dictionary of the Spanish Language]. (23. ${ }^{a}$ ed.). Madrid, Spain: Author.

[13] Gilbert, R., Thadani, V., Handy, C., Andrews, H., Sguigna, T., Sasso, A. and Payne, S. 2014. The psychological functions of avatars and alt(s): A qualitative study. Computers in Human Behavior. 32, (2014), 1-8. DOI:https://doi.org/10.1016/j.chb.2013.11.007.

[14] Gilleard, C. and Higgs, P. 2000. Cultures of Ageing Self, Citizen and the body. Pearson Education.

[15] Halliwell, E. and Dittmar, H. 2003. A Qualitative Investigation of Women 's and Men's Body Image Concerns and Their Attitudes Toward Aging. Sex Roles. 49, December (2003), 675-684.

[16] Harper, S. 2013. Future Identities: Changing identities in the UK - the next 10 years.

[17] Heart, T. and Kalderon, E. 2013. Older adults: Are they ready to adopt healthrelated ICT? International fournal of Medical Informatics. 82, 11 (2013), e209e231. DOI:https://doi.org/10.1016/j.ijmedinf.2011.03.002.

[18] Hooyman, N.R. and Kiyak, H.A. 2011. Social Gerontology: A Multidisciplinary Perspective. Pearson Education.

[19] Lindley, S.E., Harper, R. and Sellen, A. 2009. Desiring to be in touch in a changing communications landscape. Proceedings of the 27th international conference on Human factors in computing systems - CHI 09. (2009), 1693-1702. DOI:https://doi.org/10.1145/1518701.1518962.

[20] Mcarthur, V., Teather, R.J. and Jenson, J. 2015. The Avatar Affordances Framework: Mapping Affordances and Design Trends in Character Creation Interfaces. CHI Play. (2015), 231-240. DOI:https://doi.org/10.1145/2793107.2793121.

[21] McDonough, J.P. 1999. Designer Selves: Construction of Technologically Mediated Identity within Graphical, Multiuser Virtual Environments. fournal of the American Society for Information Science. 50, 10 (Aug. 1999), 855-869. DOI:https://doi.org/10.1002/(SICI)1097-4571(1999)50:10<855::AIDASI3>3.3.CO;2-Y

[22] Messner, S. 2016. What it's like to become a YouTube gaming celebrity at 80 years old. PC Gamer.

[23] Morandell, M.M., Hochgatterer, A., Fagel, S. and Wassertheurer, S. 2008 Avatars in assistive homes for the elderly a user-friendly way of interaction? Lecture Notes in Computer Science (including subseries Lecture Notes in Artificial Intelligence and Lecture Notes in Bioinformatics). 5298 LNCS, (2008), 391-402. DOI:https://doi.org/10.1007/978-3-540-89350-9-27.

[24] Mori, M. 1970. The Uncanny Valley (Translated by MacDorman, K. and Kageki, N.). IEEE Robotics and Automation Magazine. 19, 2 (1970), 98-100. DOI:https://doi.org/10.1109/MRA.2012.2192811.

[25] Neuman, W.L. 2014. Social Research Methods: Qualitative and Quantitative Approaches. Pearson Education Limited.

[26] Neustaedter, C. and Fedorovskaya, E. 2009. Presenting identity in a virtual world through avatar appearances. Proceedings of Graphics Interface 2009. (2009), 183-190.

[27] Pearce, C. 2008. The Truth About Baby Boomers Gamers: A Study of OverForty Computer Game Players. Games and Culture. 3, 2 (2008), 142-174. DOI:https://doi.org/10.1177/1555412008314132.
[28] Rice, M., Koh, R., Lui, Q., He, Q., Wan, M., Yeo, V., Ng, J. and Tan, W.P. 2013. Comparing avatar game representation preferences across three age groups. CHI '13 Extended Abstracts on Human Factors in Computing Systems on - CHI EA '13. (2013), 1161-1166. DOI:https://doi.org/10.1145/2468356.2468564.

[29] De Schutter, B. 2011. Never Too Old to Play: The Appeal of Digital Games to an Older Audience. Games and Culture. 6, 2 (2011), 155-170. DOI:https://doi.org/10.1177/1555412010364978.

[30] Sherman, S.R. 1994. Changes in age identity: Self perceptions in middle and late life. Journal of Aging Studies. 8, 4 (1994), 397-412. DOI:https://doi.org/10.1016/0890-4065(94)90011-6.

[31] Siriaraya, P., Ang, C.S. and Bobrowicz, A. 2014. Exploring the potential of virtual worlds in engaging older people and supporting healthy aging. Behaviour \& Information Technology. 33, 3 (2014), 282-293. DOI:https://doi.org/10.1080/0144929X.2012.691552.

[32] Stafford, P.B. 2015. SOCIAL MEDIA AND THE AGE-FRIENDLY COMMUNITY. Aging and the Digital Life Course. D. Garattini, Chiara Prendergast, ed. Berghahn Books. 21-38.

[33] Tiggemann, M. 2004. Body image across the adult life span: Stability and change. Body Image. 1, 1 (2004), 29-41. DOI:https://doi.org/10.1016/S17401445(03)00002-0.

[34] Timberlake, E.M. 1981. The value of grandchildren to grandmothers. Fournal of Gerontological Social Work. 3, 1 (1981), 63-76. DOI:https://doi.org/10.1300/J083V03N01.

[35] Turkle, S. 1994. Constructions and reconstructions of self in virtual reality: Playing in the MUDs. Mind, Culture, and Activity. 1, 3 (1994), 158-167. DOI:https://doi.org/10.1080/10749039409524667.

[36] Vines, J., Pritchard, G., Wright, P. and Olivier, P. 2015. An Age-Old Problem Examining the Discourses of Ageing in $\mathrm{HCI}$ and Strategies for Future Research. Tochi. 22, 1 (2015), 1-27. DOI:https://doi.org/10.1145/2696867.

[37] Waycott, J., Vetere, F., Pedell, S., Kulik, L., Ozanne, E., Gruner, A., Downs, J., Sonja, P., Kulik, L., Ozanne, E., Alan, G. and Downs, J. 2013. Older Adults as Digital Content Producers. In Proceedings of the SIGCHI conference on human factors in computing systems. ACM (2013), 39-48. DOI:https://doi.org/10.1145/2470654.2470662.

[38] Wikipedia: the Free Encyclopedia: 2017. https://en.wikipedia.org/wiki/Big Bad fohn.

[39] Wilcox, S. 1997. Age and gender in relation to body attitudes: Is there a double standard of aging? Psychology of Women Quarterly. 21, 4 (1997), 549-565. DOI:https://doi.org/10.1111/j.1471-6402.1997.tb00130.x.

[40] World Health Organization 2015. World report on Ageing And Health.

[41] Yee, N. 2014. Proteus paradox: how online games and virtual worlds change us, and how they don't. Yale University Press.

[42] Yee, N., Bailenson, J. and Rickertsen, K. 2007. A meta-analysis of the impact of the inclusion and realism of human-like faces on user experiences in interfaces. In Proceedings of the SIGCHI conference on Human factors in computing systems. ACM (2007), 1-10.

[43] Zhang, F. and Kaufman, D. 2015. Older Adults' Social Interactions in Massively Multiplayer Online Role-Playing Games (MMORPGs). Games and Culture. 11, 1-2 (2015), 150-169. DOI:https://doi.org/10.1177/1555412015601757. 


\section{University Library}

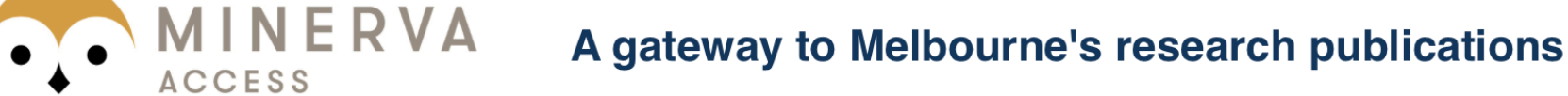

Minerva Access is the Institutional Repository of The University of Melbourne

\section{Author/s:}

Carrasco, R;Baker, S;Waycott, J;Vetere, F

Title:

Negotiating stereotypes of older adults through avatars

\section{Date:}

2017-11-28

Citation:

Carrasco, R., Baker, S., Waycott, J. \& Vetere, F. (2017). Negotiating stereotypes of older adults through avatars. Proceedings of the 29th Australian Conference on ComputerHuman Interaction, pp.218-227. Association for Computing Machinery (ACM). https:// doi.org/10.1145/3152771.3152795.

Persistent Link:

http://hdl.handle.net/11343/241955 